\title{
Pharmacotherapeutics of miglitol: an $\alpha$-glucosidase inhibitor
}

\begin{abstract}
Diabetes Mellitus (DM) is a morbid disease globally, with growing prevalence as time passes. Diabetes produces macrovascular and microvascular complications. The key cause of these complications is poorly precise postprandial hyperglycemia. Various anti-diabetic drugs are available for the management of DM and their consequences; however, they are not devoid of side effects. This mini-review highlights the important features of miglitol in term of their mechanism of actions, pharmacokinetics, adverse reactions, indication/contraindications and drug-drug interactions. Monotherapy with miglitol is well tolerated, effective in patients with hyperglycemia. Furthermore, it has been also reported that miglitol is more effective than other drugs of the $\alpha$-glucosidase inhibitors class, such as voglibose and acarbose in managing body weight gain.
\end{abstract}

Keywords: postprandial hyperglycemia, $\alpha$-glucosidase inhibitors, miglitol, drugdrug interaction
Volume 7 Issue 6 - 2018

\author{
Yogesh Kumar, Ramesh K Goyal, Ajit Kumar \\ Thakur \\ School of Pharmaceutical Sciences, Delhi Pharmaceutical \\ Sciences and Research University, India
}

Correspondence: Ajit Kumar Thakur, Assistant Professor of Pharmacology, School of Pharmaceutical Sciences, Delhi Pharmaceutical Sciences and Research University, Pushp Vihar Sector-III, M.B. Road, New Delhi-I 10 017, India, Fax +91-II29554503, Email ajitmitpharm@gmail.com

Received: June 28, 2017 | Published: November 06, 2018
Abbreviations: DM, diabetes mellitus; OHAs, oral hypoglycemic agents; GIP, glucose-dependent insulinotropic polypeptide; $\mathrm{PPHG}$, postprandial hyperglycemia; CVD, cardiovascular disease; GLP-1, glucagon like peptide-1

\section{Introduction}

Diabetes Mellitus (DM) is a chronic metabolic disorder accompanying with malfunctioning of glucose metabolism and their homeostasis. People suffering from DM showed significant morbidity and mortality due to their complications associated with microvascular and macrovascular system, and also affecting various vital organs and their functions. ${ }^{1}$ The World Health Organization (WHO) announced that the worldwide prevalence of DM in 2000 was 171 million and would reach approximately 366 million by 2030 . However, the worldwide prevalence of DM has already reached 346 million as of 2010. In a report, authors have conveyed that by the year 2030, the diabetic population in India will surge from 21.7 million to 79.4 million. ${ }^{2}$ Alpha-glucosidase inhibitors (AGIs; acarbose, miglitol, voglibose etc.) are extensively used in the management of patients with type 2 diabetes. Pharmacologically AGIs delay the absorption of carbohydrates from the small intestine and consequently have a lowering effect on postprandial blood glucose. One of the major complications to the AGIs is their side effects. Because AGIs affect carbohydrate absorption in the small intestine, they can cause bloating, nausea, diarrhea, and flatulence and sometime causes adverse effects also. The aim of this mini-review to give the comparative overview on AGIs, as well as pharmacotherapy and their potential role of miglitol for the management of postprandial hyperglycemia and cardiovascular surrogates in patients with type- 2 diabetes mellitus.

\section{Pharmacotherapeutics of miglitol}

Alpha glucosidase inhibitors are a unique class of anti-diabetic drugs. Derived from bacteria, these oral drugs are enzyme inhibitors which do not have a pancreato -centered mechanism of action. Working to delay carbohydrate absorption in the gastrointestinal tract, they control postprandial hyperglycemia. Acarbose was first developed in Germany and voglibose is a Japanese invention while miglitol is an American discovery. ${ }^{3}$ Acarbose and voglibose are not absorbed from the intestine and have poor bioavailability. Miglitol, on the contrary, is almost completely absorbed from the upper part of the intestine. ${ }^{3}$ Miglitol is a new glucosidase inhibitor that is absorbed rapidly and almost completely from the small intestine after oral administration, consequently suppressing postprandial blood glucose levels more effectively than other $\alpha$-glucosidase inhibitors. ${ }^{4}$

In a study, authors have revealed that the subjects with impaired glucose tolerance demonstrated reduced postprandial hyperglycemia after the treatments with $\alpha$-glucosidase inhibitor. Furthermore, they warranted that $\alpha$-glucosidase inhibitor not only causes reduction in the risk of progression to DM, but also reduction in development of new cases of hypertension and the risk of cardiovascular disease. ${ }^{1}$ Previous studies suggest that miglitol improves the release of GLP-1 whereas it suppresses the release of glucose-dependent insulinotropic polypeptide (GIP) by increasing glucose absorption from the lower small intestine. ${ }^{5}$ Both $\alpha$-glucosidase inhibitors, acarbose and miglitol are effective in lowering PPBS as well as HbAlc, but efficacy and tolerability (Table 1) of miglitol was proven to be better than acarbose. ${ }^{6}$

In this mini-review, we have highlighted the various effects miglitol, and $\alpha$-glucosidase inhibitors class of drug on type- 2 diabetes mellitus, on lipid profile, on obesity and body weight and effects of miglitol on cardiovascular disease surrogates. Miglitol is the first pseudo monosaccharide $\alpha$-glucosidase inhibitor derived from 1deoxynojirimycin and is structurally glucose analog (Figure 1). ${ }^{7}$

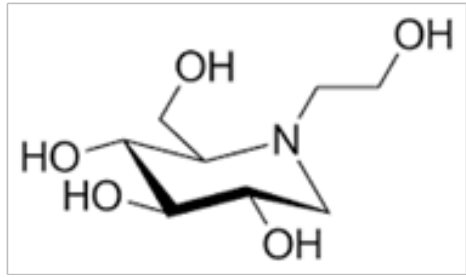

Figure I Structure of miglitol.

\section{Mechanism of action}

The therapeutic effect of miglitol results from a reversible inhibition of $\alpha$-glucosidase enzymes, an intestinal membrane 
bound hydrolyze enzyme, which hydrolyzes oligosaccharides and disaccharides to glucose and other monosaccharides in the brush border of the small intestine (Figure 2). This results in a reduction in postprandial hyperglycemia. ${ }^{8}$

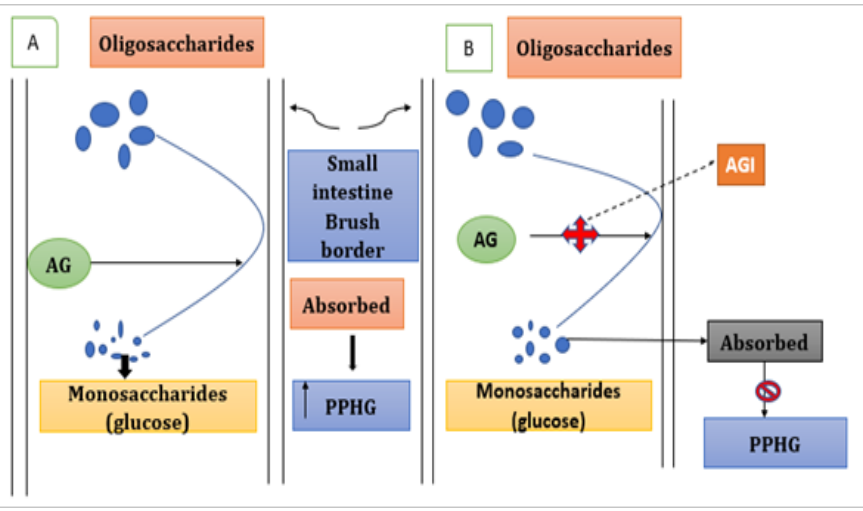

Figure 2 Mechanism of action of miglitol. In part A, the oligosaccharides in the present of $\alpha$-glucosidase enzyme converted into monosaccharides and increased postprandial glucose level. Part B indicate that $\alpha$-glucosidase inhibitors inhibit the enzyme and not allowed to conversion of oligosaccharides into monosaccharides and reduced postprandial hyperglycaemia.

Table I Comparative efficacy and safety of $\alpha$-glucosidase inhibitors ${ }^{7}$

\begin{tabular}{lll}
\hline S. no & Comparison & a-glucosidase inhibitors \\
\hline I. & HbAlc & Favored miglitol (high) \\
2. & Weight & Favored miglitol (high) \\
3. & LDL & Favored acarbose (moderate) \\
4. & HDL & Neither favored \\
5. & TG & Neither favored \\
6. & Hypoglycemia & Favored miglitol (high)
\end{tabular}

Abbreviations: LDL, low density lipoprotein; HDL, high density lipoprotein; TG, triglycerides

\section{Pharmacological action of miglitol}

Effect on cardiovascular system: Hyperglycemia is the risk factor for progression of cardiovascular disease and causes endothelial dysfunction. Miglitol improves postprandial hyperglycemia (PPHG) and this may produce therapeutic effects on associated cardiovascular disease. Miglitol potential to reduce cardiovascular disease (CVD) by direct virtue of its mode of action by reducing the postprandial hyperglycemia, glucose variability and (reactive) hypoglycemia, increasing the insulin sensitivity, enhancing the glucagon like peptide-1(GLP-1) release more significantly as compare to acarbose and voglibose, impacting on and synergizes with gut microbiota and induction of moderate weight loss is better with miglitol. Miglitol also reduce CVD by effects on CVD surrogate outcomes by restoration of endothelial function, reduction of microalbuminuria and nephropathy, reduction of carotid intima-media thickening and reduction of oxidative stress.

\section{Clinical advantages}

Miglitol is generally used as therapeutic agents for the management of hyperglycemia, when oral hypoglycemic agents (OHAs) or insulin preparations, in addition to diet and/or exercise, do not result in an adequate glycemic control. Thus, miglitol is indicated in:

Patients with NIDDM: Miglitol is indicated to the patients with
NIDDM in addition to their balance diet and exercise to improve glycemic control, where normoglycemia cannot be achieved by diet alone. ${ }^{9,10}$

Combination with other OHAs: Miglitol along with metformin or anagliptin provides a synergistic effect on glycemic control, as indicated by the noticeable reductions in $\mathrm{HbA} 1 \mathrm{c}$ and plasma glucose levels in middle-aged patients in whom type-2 diabetes is insufficiently controlled by dietary management. ${ }^{11}$

Prevention of type-2 diabetes: In a study, Hsieh et al. ${ }^{12}$ have showed that miglitol significantly improves metabolic control in Chinese patients with type- 2 diabetes mellitus. ${ }^{12}$ Miglitol is safe and well tolerated drug with the exclusion of abdominal discomfort. ${ }^{13}$ Therefore, miglitol may be a useful as an adjuvant therapy for patients with type- 2 diabetes mellitus ineffectively controlled by diet and sulfonylurea treatment. ${ }^{12}$

Effects on lipid profile: It has been reported that, patients suffering from type-2 diabetes have a comorbid dyslipidemia that increases risk of CVD. The study of Segal et al. ${ }^{10}$ showed that there are no significant effects of miglitol on triglycerides and total cholesterol were observed. Due to inconclusive data, miglitol had no consistent significant effect on lipid component. ${ }^{14}$

Effect on obesity and body weight: Four clinical trials that carried from 2012 to 2014 in Japan, obviously exhibiting that miglitol demonstrates an anti-obesity effect. In each of the four studies, miglitol essentially diminished the body weight and weight file of obese or type-2 diabetic patients. According to the study Giuseppe Derosa et al. ${ }^{13}$ showed that combination of miglitol and metformin shows better result to control $\mathrm{HbA} 1 \mathrm{c}$, PPG and body weight. ${ }^{13}$ Miglitol is more effective than other drugs of $\alpha$-glucosidase inhibitors such as voglibose and acarbose in managing body weight gain. ${ }^{13}$

Pharmacokinetics: Miglitol is absorbed by the active transport mechanism and its rate of absorption is limited. Due to this mechanism bioavailability is variable in the drug. ${ }^{14}$ When the miglitol is given at the dose of $25 \mathrm{mg}$, its shows $100 \%$ bioavailability but when it is given at a higher dose its shows 50 to $70 \%$ bioavailability. Peak plasma concentration of the drug comes into 2-3 hours. The volume of distribution of the drug is small in the body ${ }^{15}$ which indicates that miglitol is distributed into extracellular fluids only and have low tissue penetration. Protein binding of the drug is almost negligible $<4 \%$ and permeation of drug from the blood brain barrier is in low amount. The miglitol is excreted in unchanged form in the urine or feces. The amount of drug excreted is depend on the amount of the drug is absorbed by the small intestine. The elimination half-life of miglitol is about 2 to 3 hours. ${ }^{16}$

Adverse reactions: The most common adverse effects of the drug miglitol is gastrointestinal and directly related to the mechanism of drug. The most common adverse effects with the miglitol drug such as: flatulence, nausea, abdominal pain or distention, diarrhea. ${ }^{17}$

Contraindication and drug interactions: Miglitol is contraindicated in the patients having disease like diabetic ketoacidosis, gastrointestinal complications such as colonic ulceration or partial intestinal obstruction, inflammatory bowel disease, obstructive intestinal disease, chronic intestinal disease with marked disorders of absorption. ${ }^{8}$ Miglitol should not be use in the women who are breastfeeding. ${ }^{16}$ Miglitol are interacted with various drugs that discussed in the Table 2. 
Table 2 Tabulated summary of following drug interaction with miglitol

\begin{tabular}{llll}
\hline S. no. & Drug & Interaction & Reference \\
\hline I & Phenytoin & Shift in the time of peak concentration by approximately $10-15 \%$ & Plo \\
2 & Tofogliflozin & Plasma concentration time profiles identical and similar rate and amount of absorption. & 19 \\
3 & Glibenclamide & Lowered the peak concentration and amount of absorption & 16 \\
4 & Warfarin & No significant effect on pharmacokinetics & 16 \\
5 & Metformin & Lowered the peak concentration and amount of absorption & 16 \\
6 & Digoxin & Average plasma concentration reduced & 20
\end{tabular}

\section{Conclusion}

Thus, miglitol possibly offers alternative pharmaco-therapeutic choice for the patients suffering from type-2 diabetes mellitus with poor glycemic control. Additionally, miglitol may deferred the need of beginning of pharmaco-therapy to the patients suffering from type-2 diabetes. Miglitol improves the postprandial blood glucose level in NIDDM patients who are poorly maintained, in spite of diet control and pharmacological therapy. Safety study for miglitol in diabetic patients have been explored and found harmless, on the other hand it is contraindicated in the patients having disease like diabetic ketoacidosis, gastrointestinal complications and not to be used in breast feeding women. Thus, miglitol is safe and effective drug in the prevention of type- 2 diabetes. According to the previous studies, it has been concluded that miglitol is the preferable agent for improving glycemic control with promoting bodyweight reduction. Several reports suggested that miglitol increases GLP-1 and decreases GIP to greater extents than voglibose after a single or long-term administration in type 2 diabetes. Therefore, pharmacotherapy with miglitol may be an effective strategy in the treatment and management of postprandial hyperglycemia and cardiovascular surrogates in patients with type-2 diabetes mellitus.

\section{Acknowledgements}

None.

\section{Conflict of interest}

The author declares that there is no conflict of interest.

\section{References}

1. Dabhi AS, Bhatt NR, Shah MJ. Voglibose: An Alpha Glucosidase Inhibitor J Clin Diagn Res. 2013;7(12):3023-3027.

2. Joung KH, Jeong JW, Ku BJ. The association between type 2 diabetes mellitus and women cancer: the epidemiological evidences and putative mechanisms. Biomed Res Int. 2015:920618.

3. Kalra S. Alpha glucosidase Inhibitors. J Pak Med Assoc. 2014;64(4):474 476 .

4. Emoto T, Sawada T, Hashimoto M, et al. Effect of 3-month repeated administration of miglitol on vascular endothelial function in patients with diabetes mellitus and coronary artery disease. Am J Cardiol. 2012;109(1):42-46.

5. Lee A, Patrick P, Wishart J, et al. The effects of miglitol on glucagonlike peptide-1 secretion and appetite sensations in obese type 2 diabetics. Diabetes Obes Metab. 2002;4(5):329-335.
6. Choudhury LD, Basu R, Biswas T, et al. A comparative study of miglitol and acarbose add on therapy intended for better glycaemic control in type 2 diabetes mellitus. Int J Cur Res Rev. 2016;24(8):33-40.

7. Sugihara H, Nagao M, Harada T, et al. Comparison of three $\alpha$-glucosidase inhibitors for glycemic control and bodyweight reduction in Japanese patients with obese type 2 diabetes. J Diabetes Invest. 2004;5(2):206-212.

8. Derosa G, Maffioli P. $\alpha$-Glucosidase inhibitors and their use in clinical practice. Arch Med Sci. 2012;8(5):899-906.

9. Kingma PJ, Menheere PP, Sels JP, et al. $\alpha$-Glucosidase inhibition by miglitol in NIDDM patients. Diabetes Care. 1992;15(4):478-483.

10. Segal P, Feig PU, Schernthaner G. The efficacy and safety of miglitol therapy compared with glibenclamide in patients with NIDDM inadequately controlled by diet alone. Diabetes care. 1997;20(5):687-691.

11. Chiasson JL, Naditch L. The synergistic effect of miglitol plus metformin combination therapy in the treatment of type 2 diabetes. Diabetes Care. 2001;24(6):989-994.

12. Hsieh SH, Shih $\mathrm{KC}$, Chou $\mathrm{CW}$, et al. Evaluation of the efficacy and tolerability of miglitol in Chinese patients with type 2 diabetes mellitus inadequately controlled by diet and sulfonylureas. Acta Diabetol. 2011;48(1):71-77.

13. Sugimoto S, Nakajima H, Kosaka K, et al. Review: Miglitol has potential as a therapeutic drug against obesity. Nutr Metab (Lond). 2015;12(1):51.

14. Buse JB, Tan MH, Prince MJ, et al. The effects of oral anti-hyperglycaemic medications on serum lipid profiles in patients with type 2 diabetes. Diabetes Obes Metab. 2004;6(2):133-156.

15. Nomura S, Omoto S, Yokoi T, et al. Effects of miglitol in platelet-derived microparticle, adiponectin and selectin level in patients with type 2 diabetes mellitus. Int J Gen Med. 2011;4:539-545.

16. Campbell LK, Baker DE, Campbell RK. Miglitol: assessment of its role in the treatment of patients with diabetes mellitus. Ann Pharmacother. 2000;34(11):1291-1301.

17. Johnston PS, Feig PU, Coniff Rf, et al. Long-term titrated-dose alphaglucosidase inhibition in non-insulin-requiring Hispanic NIDDM patients. Diabetes Care. 1998;21(3):409-415.

18. Ratanakorn D, Kaojarern S, Phuapradit P, et al. Single oral loading dose of phenytoin: a pharmacokinetics study. J Neurol Sci. 1997;147(1):89-92.

19. Kasahara N, Fukase H, Ohba Y, et al. A Pharmacokinetic/Pharmacodynamic Drug-Drug Interaction Study of Tofogliflozin (a New SGLT2 inhibitor) and Selected Anti-Type 2 Diabetes Mellitus Drugs. Drug Res (Stuttg). 2016;66(2):74-81.

20. Effects of drug-drug interactions involving oral antihyperglycaemics are, with the exception of hypoglycaemia, mostly clinically unimportant. Drugs \& Therapy Perspectives. 2006;22(4):18-22. 